\title{
Electron Supersurface Scattering On Polycrystalline Au
}

\author{
Wolfgang S. M. Werner* \\ Institute of Applied Physics, Vienna University of Technology, Wiedner Hauptstraße 8-10, A 1040 Vienna, Austria
}

Mihály Novák ${ }^{\dagger}$

Université Libre de Bruxelles, Service de Métrologie Nucléaire (CP 165/84), 50 avenue F. D. Roosevelt, B-1050 Brussels, Belgium

Francesc Salvat-Pujol

Institut für Theoretische Physik, J.W.Goethe-Universität Frankfurt, Max-von-Laue-Straße 1, 60438 Frankfurt am Main, Germany

Josef Zemek and Petr Jiricek

Institute of Physics, Academy of Sciences of the Czech Republic, Na Slovance 2, 18221 Prague 8, Czech Republic

(Received 10 August 2012; revised manuscript received 20 December 2012; published 22 February 2013)

\begin{abstract}
Supersurface electron scattering, i.e., electron energy losses and associated deflections in vacuum above the surface of a medium, is shown to contribute significantly to electron spectra. We have obtained experimental verification (in absolute units) of theoretical predictions that the angular distribution of the supersurface backscattering probability exhibits strong oscillations which are anticorrelated with the generalized Ramsauer-Townsend minima in the backscattering probability. We have investigated 500-eV electron backscattering from an Au surface for an incidence angle of $70^{\circ}$ and scattering angles between $37^{\circ}$ and $165^{\circ}$. After removing the contribution of supersurface scattering from the experimental data, the resulting angular and energy distribution agrees with the Landau-Goudsmit-Saunderson (LGS) theory, which was proposed about 60 years ago, while the raw data are anticorrelated with LGS theory. This result implies that supersurface scattering is an essential phenomenon for quantitative understanding of electron spectra.
\end{abstract}

DOI: 10.1103/PhysRevLett.110.086110

PACS numbers: 68.49.Jk, 79.20.-m, 79.60.-i

Electron scattering from solid surfaces has been of paramount importance in fundamental and applied science and technology ever since the first experiments, conducted over a century ago [1]. With the advent of nanotechnology, the interest in the details of the dynamics of electrons near a vacuum-solid boundary has seen a remarkable revival. In particular, so-called "near-field" electron energy-loss spectroscopy [2-5] has been established as an effective tool to probe electro-optical modes of the solid-state electrons in nanostructured surfaces. Furthermore, newly developing electron-beam techniques such as the near field emission secondary electron microscope [6], need an increasingly refined model of the electron-surface interaction. While near-field energy loss spectroscopy in transmission is nowadays well established, observing supersurface scattering in a reflection geometry poses a great experimental challenge, since the resulting spectra are dominated by multiple losses experienced inside the solid.

Model calculations $[7,8]$ predict that for scattering geometries near the very sharp Ramsauer-Townsend minima in the elastic-scattering cross section, the contribution of

Published by the American Physical Society under the terms of the Creative Commons Attribution 3.0 License. Further distribution of this work must maintain attribution to the author(s) and the published article's title, journal citation, and DOI. the supersurface inelastic-scattering probability in reflection electron energy-loss spectra (REELS) is significantly enhanced by the (small-angle) deflections accompanying a supersurface energy transfer, leading to oscillatory structures in the angular distribution of the surface-excitation probability. This effect makes unambiguous identification of supersurface scattering in a reflection experiment possible and elucidates the role of small-angle deflections taking place during supersurface electron scattering.

Figure 1 illustrates the supersurface scattering process for $500-\mathrm{eV}$ electrons backscattered from a $\mathrm{Au}$ surface. Panel (a) shows the situation without deflection; panel (b) is for a finite supersurface scattering deflection angle $\theta_{\text {ss }}$ before elastic backscattering, and panel (c) after elastic backscattering. The backward part of the differential elastic cross section (DECS) is illustrated in Fig. 1 in a polar representation in blue [shown in full in Fig. 2(a) as the green curve]. The DECS exhibits deep minima at scattering angles of $65^{\circ}, 110^{\circ}$, and $153^{\circ}$, the so-called RamsauerTownsend minima $[9,10]$ that reflect the destructive interference of partial waves with a given angular momentum which experience different phase shifts during their passage through the screened Coulomb potential of the nuclei in the target. All panels in Fig. 1 are for the same incidence $\left(\theta_{i}\right)$ and emission $\left(\theta_{o}\right)$ angles corresponding to a net deflection angle $\left(\theta_{s}\right)$ for which the DECS exhibits a deep minimum. 


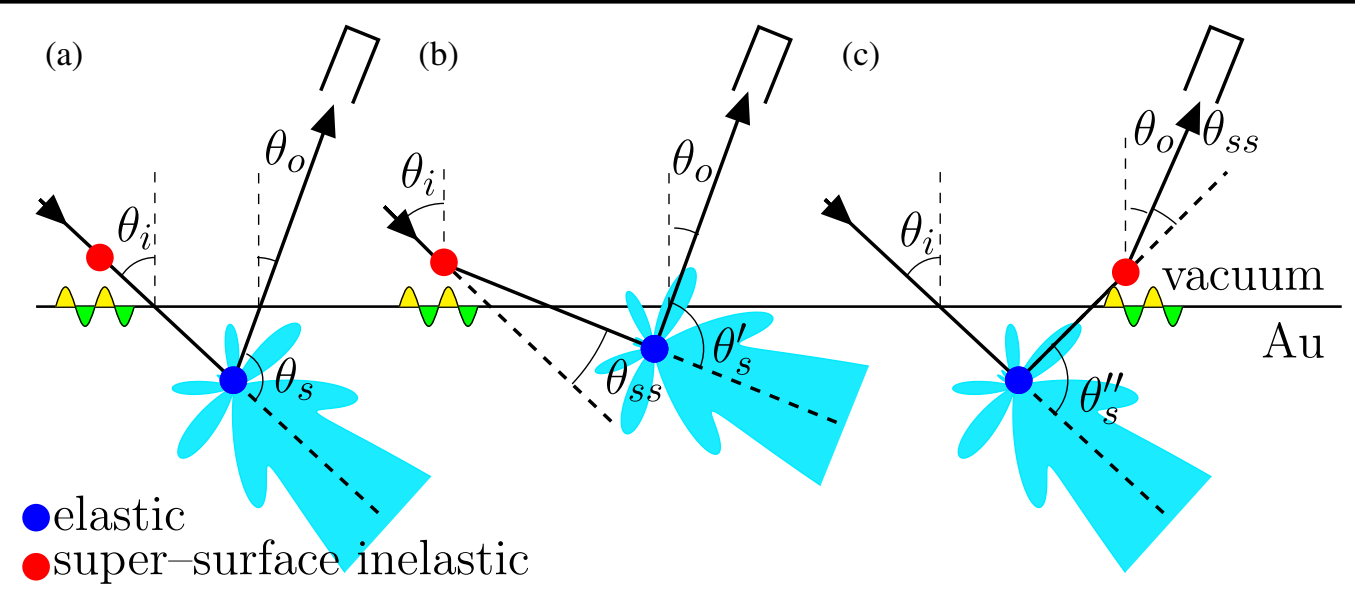

FIG. 1 (color online). Illustration of supersurface scattering: (a) without deflections during surface excitations; surface excitation before (b) and after (c) backscattering with associated deflections $\left(\theta_{\mathrm{ss}}\right)$ (see text).

As illustrated in Fig. 1(b), during the approach to the surface, while still in vacuum, an electron may polarize the surface charge, leading to a supersurface energy loss in which a small amount of momentum is transferred, giving rise to a deflection $\left(\left\langle\theta_{\mathrm{ss}}\right\rangle \approx 8^{\circ}\right.$, for $500-\mathrm{eV}$ electrons on $\mathrm{Au}$ on a binary collision model). After a supersurface interaction, the scattering angle $\theta_{s}^{\prime}$ which is then relevant for the net deflection is no longer exactly at the minimum of the DECS. In other words, the reflection probability increases significantly when the incoming electron experiences a deflection in a supersurface collision since, in the vicinity of a minimum, the elastic cross section increases rapidly with the scattering angle [see the inset of Fig. 2(a)]. Of course, the opposite effect, in which an electron is scattered out of a maximum in the DECS in the course of a supersurface collision, also takes place, but the point is that the maxima in the DECS are quite broad compared to the minima; therefore, a net effect is only expected near a minimum in the DECS.

Supersurface scattering not only has a measurable effect if it takes place before the electron enters the solid, but also after it leaves the solid. Consider the situation depicted in Fig. 1(c): after elastic backscattering over $\theta_{s}^{\prime \prime}$, a large fraction of electrons will have a direction near the scattering maximum at $\sim 90^{\circ}$, and will therefore be outside the detection cone. However, after experiencing a small angle deflection $\theta_{\mathrm{ss}}$ in the course of a supersurface excitation, some of them are scattered into the detector. Again, the opposite effect (scattering out of the detector) also takes place but the point is that this scattering concerns a very small fraction of electrons leaving the solid after elastic backscattering, since the detector direction coincides with a minimum in the elastic-scattering cross section.

A comparison of Monte Carlo model calculations and experimental results for the angular distribution of $500-\mathrm{eV}$ electrons elastically backscattered from a polycrystalline $\mathrm{Au}$ surface is presented in Fig. 2, for an incidence angle of $70^{\circ}$. The angular distribution of the elastically backscattered intensity in the plane of incidence is presented in Fig. 2(a). In Fig. 2(b), the average number of surface excitations experienced inside the solid is represented by the lower (blue) curves; the upper (red) curves correspond to supersurface scattering. Dashed curves are without deflection in an inelastic process, solid curves are for finite scattering angles $\theta_{\mathrm{ss}}$, calculated on a binary collision model [8]. The experimental results are represented by the black solid curves with data points. Details concerning the model calculations and the experimental procedure can be found in Refs. [7,8,11].

Figure 2(a) compares the angular distribution of the elastic reflection coefficient with the DECS. The minima in the experimental data points are less deep, which is caused on one hand by the finite opening angle of the analyzer and, on the other hand, by multiple elastic scattering inside the target. Goudsmit and Saunderson already stipulated [12] that the angular distribution after multiple elastic scattering can be conceived as a multiple selfconvolution of the DECS and becomes nearly isotropic after a few elastic collisions $\left(n_{e} \sim 4-5\right)$. The analytic model [red curve in Fig. 2(a)] of Oswald, Kasper, and Gaukler (OKG), which is based on this idea [13,14], is indeed seen to reproduce the experimental data quite well and is in close agreement with Monte Carlo model calculations (blue dashed curve). The deviations for emission angles $\left|\theta_{o}\right|>50^{\circ}$ are attributed to a slight experimental misalignment. The experimental data were normalized to the OKG results at $\theta_{s}=80^{\circ}$.

According to the common model for the angular distribution of the surface excitation probability [15], a smooth dependence following the reciprocal cosine of the emission angle is expected for the angular dependence. The upper (red) dashed curve in Fig. 2(b) approximately follows this law, except for emission angles near minima in the cross section. This minor effect is caused by the energy 


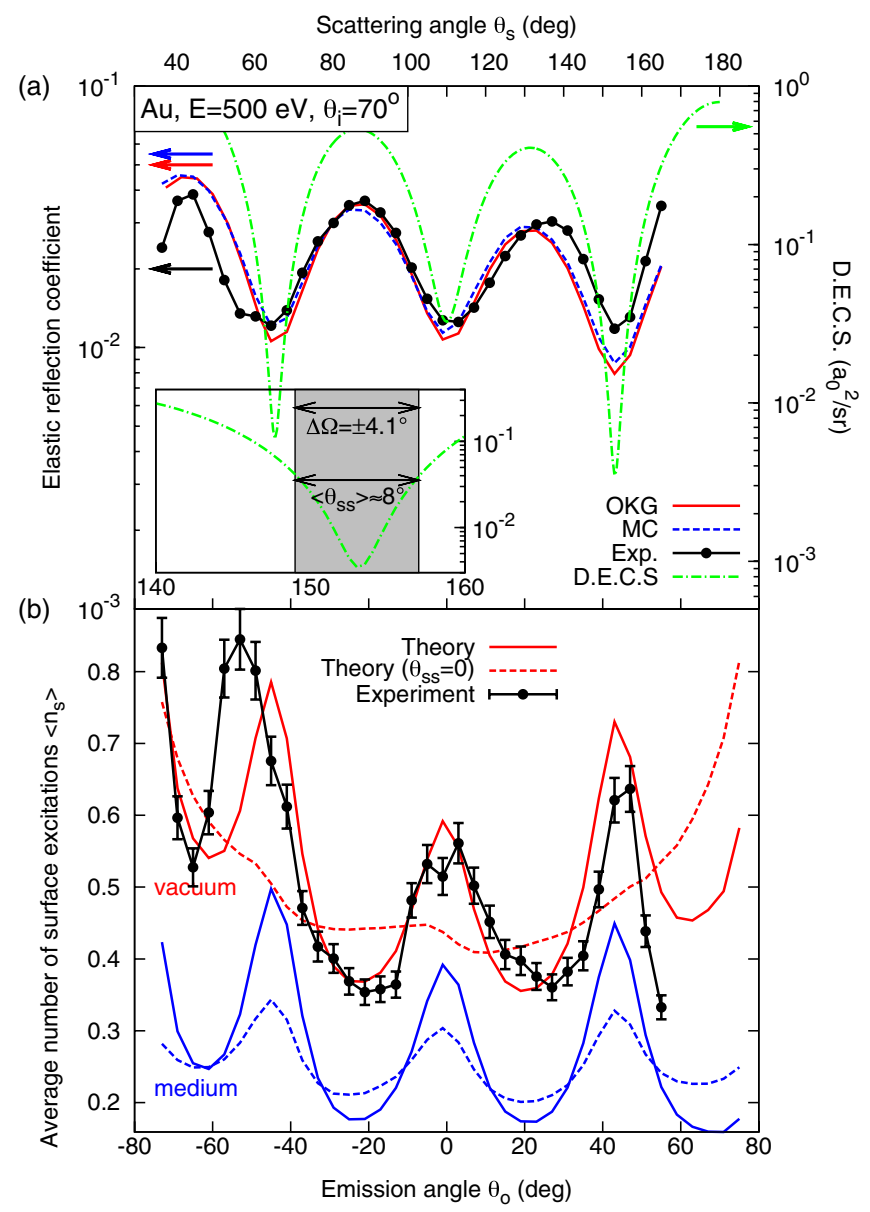

FIG. 2 (color online). (a) Solid curves: elastic peak intensity according to the Oswald-Kasper-Gaukler (OKG)-model [13]; Dashed curve: Monte Carlo (MC) calculations. Data points with solid curves: measured elastic peak intensity normalised to theory at a scattering angle of $80^{\circ}$; Dash-dotted curves: differential elastic-scattering cross section (DECS). The inset compares an expanded view of the DECS for $140^{\circ}<\theta_{s}<160^{\circ}$ with the solid angle of the analyzer and the average deflection angle in a supersurface process. (b) Solid curves with data points: average number of surface excitations derived from experiment. Dashed and solid curves: Monte Carlo calculations with (solid curves) and without (dashed curves) deflections during inelastic scattering. Lower curves: surface excitations taking place inside the medium; Upper curves: surface excitations in vacuum; Note that this comparison is in absolute units.

dependence of the elastic-scattering cross section. In particular, the position of the minima in the DECS shifts by a few degrees when the probing electron loses about $10 \%$ of its initial energy. Since the DECS varies rapidly with the scattering angle near a deep minimum, an energy loss experienced along the electron's trajectory may significantly enhance the probability for it to be reflected compared to the quasielastic case where the position of the minima in the DECS is taken to be independent of the energy loss. This effect is of course stronger when the electron is inside the medium since there it cannot only experience surface excitations (with comparatively small energy losses), but also (multiple) volume excitations and in addition suffer a surface loss on its way out. When deflections in inelastic collisions come into play, both the vacuum and medium contribution of the surface excitation probability (solid curves) exhibit strong oscillations which are anticorrelated with the oscillations observed in the elastic backscattering probability [Fig. 2(a)] and provide a clear signature of supersurface scattering in a reflection experiment.

It is assumed by many authors that surface excitations experienced inside the solid are not observed in experimental loss spectra since they are compensated by a corresponding decrease of volume excitations, the essence of the so-called begrenzungs effect [16-18]. The present results for the surface excitations, shown as solid circles with error bars in Fig. 2(b), confirm this point of view in that the data agree quite well with the supersurface contribution predicted by the model calculations: apart from features in the angular distribution for $\left|\theta_{o}\right|>50^{\circ}$ the agreement is within a few percent in absolute units.

The agreement between the present experimental results and the model calculations accounting in detail for the surface dielectric response of the solid-state electrons [7] has some interesting consequences. First of all, although the existence of energy losses in supersurface scattering is well established [2-4], the present findings demonstrate that deflections during supersurface collisions need also to be taken into account when the dynamics of electrons near surfaces are investigated in detail. It is found that such deflections may in a first approximation be modeled classically. Second, the fact that the surface excitation probabilities derived from experimental loss spectra agree in absolute units with theoretical predictions for the vacuum contribution [solid red curve in Fig. 2(b)] shows that, by virtue of the begrenzungs effect, surface losses observed in energy loss experiments are predominantly attributable to supersurface scattering. Inside the medium, the occurrence of surface losses is compensated by a decrease of the bulk modes of the polarization of the solid-state electrons.

Another interesting question is the role of supersurface scattering in multiple electron scattering in non-crystalline solids. The state-of-the-art model for multiple electron scattering is provided by the Landau-Goudsmit-Saunderson (LGS) loss function [12,14,19]

$$
G(s, T, \vec{\Omega})=\sum_{n_{i}=0}^{\infty} W_{n_{i}}(s) \Gamma_{n_{i}}(T) \sum_{n_{e}=0}^{\infty} \gamma_{n_{e}} W_{n_{e}}(s) \Gamma_{n_{e}}(\vec{\Omega}),
$$

which describes the distribution of energy losses $T$ as a function of the pathlength $s$ traveled in the medium for a scattering geometry described by $\vec{\Omega}$. The subscripts " $e$ " and " $i$ " indicate elastic and inelastic scattering, respectively; the functions $W_{n}(s)$ represent the Poisson statistical process; and the quantities $\Gamma_{n}$ are multiple self-convolutions of the (in)-elastic inverse mean free paths. The first factor in this 


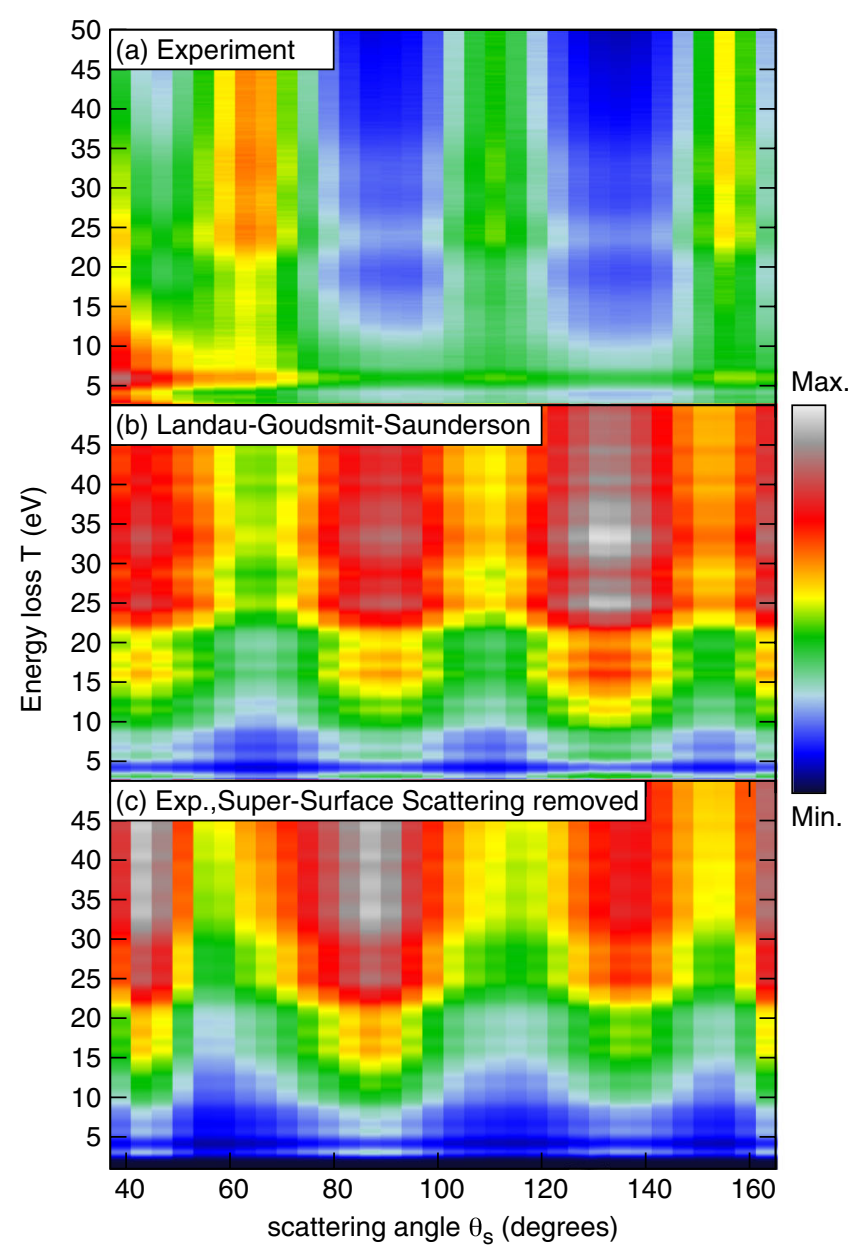

FIG. 3 (color online). Combined energy and angular spectrum of $500 \mathrm{eV}$ electrons backscattered from an Au surface. The elastic peak has been removed from all data sets to improve contrast. (a) As measured. (b) Theoretical results according to the LandauGoudsmit-Saunderson theory $[12,14,19]$. (c) Experimental data after elimination of the supersurface scattering contribution.

equation is Landau's celebrated loss function [19], written in an inelastic collision expansion [14], the second sum is the distribution of pathlengths derived for an infinite medium by Goudsmit and Saunderson [12], modified to account for arbitrary boundary conditions by means of the coefficients $\gamma_{n_{e}}$. A numerical evaluation of this expression pertaining to the present experiment is given in Fig. 3(b), and is compared with the raw experimental data in Fig. 3(a). The resulting combined energy-angular distributions are not only essentially different along the surface scattering ridge at an energy loss of $\sim 5 \mathrm{eV}$, which was to be expected, but also all remaining features are essentially anticorrelated, just as in Figs. 2(b) and 2(c) for the surface excitation probability and the elastic backscattering probability.

However, when the contribution of supersurface scattering is removed from the raw data [8], the resulting spectra, shown in Fig. 3(c), are essentially in agreement with the LGS theory. The minor differences between the relative

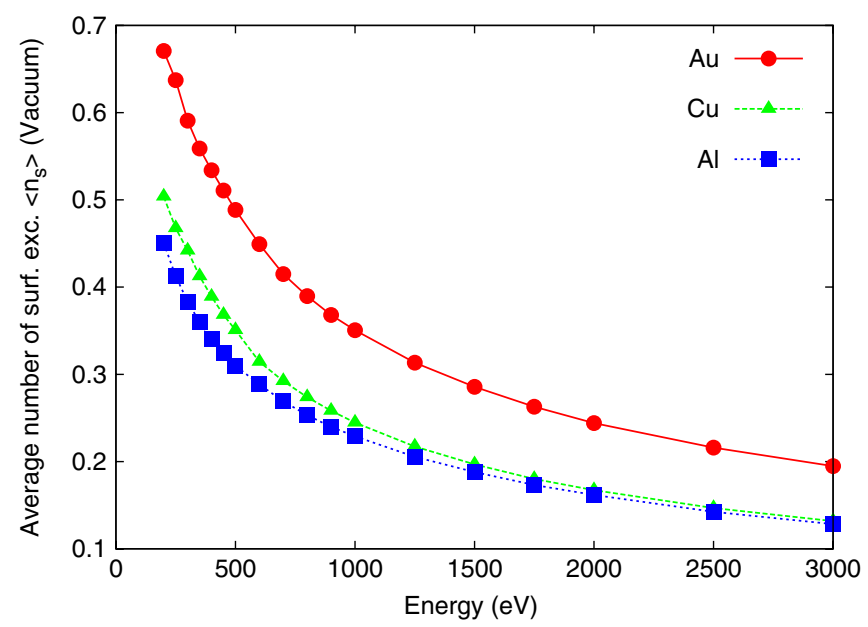

FIG. 4 (color online). Average number of surface excitations in vacuum (per surface crossing) for $\mathrm{Au}, \mathrm{Cu}$, and $\mathrm{Al}$ for energies between 200 and $3000 \mathrm{eV}$, and an incidence angle of $60^{\circ}$. These results were obtained by averaging the angular distribution in the plane of incidence for emission angles between $-60^{\circ}$ and $60^{\circ}$.

intensities of the maxima at $\theta_{s}=41^{\circ}, 87^{\circ}$, and $131^{\circ}$ in Figs. 3(b) and 3(c) are of the same order of magnitude as in Figs. 2(a) and 2(b) and are attributed to experimental misalignment. Thus, by appropriately accounting for supersurface scattering, it is now finally possible, also in the case of electron spectra, to unambiguously expose the effects of multiple scattering inside solids as described by LGS theory that was introduced more than fifty years ago.

An impression of the extent of supersurface scattering in electron spectra in general is presented in Fig. 4. Assuming the Poisson stochastic process to govern plural surface scattering [20], the reduction in the elastic intensity (in, e.g., photoelectron spectra) varies between $\sim 50 \%$ for $300 \mathrm{eV}$ and still assumes appreciable values of $\sim 10 \%$ at $3 \mathrm{keV}$.

Throughout the present work a somewhat special situation has been considered in order to obtain clear information about the supersurface scattering process. However, the complementarity between supersurface scattering and scattering inside the medium seen in Fig. 3, makes it obvious that a consistent description of any electron beam experiment involving solid-vacuum boundaries needs to go beyond the traditional description in terms of the elastic and inelastic backscattering coefficient and the secondary electron yield. In the case of inelastic backscattering a distinction should be made between the volume inelastic backscattering coefficient and a novel physical quantity, the supersurface inelastic backscattering coefficient.

This work was supported by the Austrian Science Foundation FWF (Project P20891-N20), the Czech Science Foundation (Grant No. 104/09/1269), and the Hungarian Grant OTKA 67873, which are gratefully acknowledged by the authors. F. S-P. would like to express his gratitude to the Fundación Caja Madrid for financial support and a travel grant of Fundació Agustí Pedro i Pons. 
*werner@iap.tuwien.ac.at

'On leave from Institute of Nuclear Research of the Hungarian Academy of Sciences (MTA ATOMKI), 18/c Bem tér, H-4026 Debrecen, Hungary.

[1] H. Starke, Ann. Phys. (N.Y.) 66, 49 (1898).

[2] L. D. Marks, Solid State Commun. 43, 727 (1982).

[3] T. L. Ferrell and P. M. Echenique, Phys. Rev. Lett. 55, 1526 (1985).

[4] H. Cohen, T. Maniv, R. Tenne, Y. R. Hacohen, O. Stephan, and C. Colliex, Phys. Rev. Lett. 80, 782 (1998).

[5] F. J. Garcia de Abajo, Rev. Mod. Phys. 82, 209 (2010).

[6] T. L. Kirk, O. Scholder, L. G. D. Pietro, U. Ramsperger, and D. Pescia, Appl. Phys. Lett. 94, 153502 (2009).

[7] F. Salvat-Pujol and W. S. M. Werner, Surf. Interface Anal., doi:10.1002/sia.5175 (2012).

[8] See Supplemental Material at http://link.aps.org/ supplemental/10.1103/PhysRevLett.110.086110 for details of the experimental, data analysis and simulation procedure.
[9] C. Ramsauer, Ann. Phys. (Berlin) 64, 521 (1921).

[10] P. D. Townsend and P. Bailey, Philos. Mag. 43, 593 (1922).

[11] W. S. M. Werner, J. Zemek, and P. Jiricek, Phys. Rev. B 82, 155422 (2010).

[12] S. Goudsmit and J. L. Saunderson, Phys. Rev. 57, 24 (1940).

[13] R. Oswald, E. Kasper, and K. Gaukler, J. Electron Spectrosc. Relat. Phenom. 61, 251 (1993).

[14] W. S. M. Werner, Phys. Rev. B 71, 115415 (2005).

[15] W. S. M. Werner, W. Smekal, F. Salvat-Pujol, Z. Halavani, S. Pfleger, J. Rastl, and C. Eisenmenger-Sittner, Appl. Phys. Lett. 98, 193111 (2011).

[16] R. H. Ritchie, Phys. Rev. 106, 874 (1957).

[17] C. J. Tung, Y. F. Chen, C. M. Kwei, and T. L. Chou, Phys. Rev. B 49, 16684 (1994).

[18] W. S. M. Werner, Surf. Sci. 526, L159 (2003).

[19] L. D. Landau, J. Phys. (Moscow) 8, 201 (1944).

[20] W. S. M. Werner, Phys. Rev. B 74, 075421 (2006). 